\title{
A importância do conhecimento da Síndrome de Charles Bonnet pelo médico oftalmologista e psiquiatra
}

\author{
The importance of the knowledge of the Charles Bonnet Syndrome by the ophthalmologist \\ and psychiatrist
}

\author{
Luiz Guilherme de Moraes Matheus ${ }^{1}$, Bernardo Kaplan Moscovici ${ }^{2}$, Robson André $\mathrm{Jr}^{3}$, Nayara Marques \\ Zahr ${ }^{1}$, Elis Marina Martinelli Guelfi ${ }^{1}$, Karen Asano Paiva Magalhães ${ }^{1}$, Carolina Pontes Lima ${ }^{1}$
}

\begin{abstract}
Resumo
Introdução: A sindrome de Charles Bonnet é definida como a percepção de alucinações visuais complexas em pacientes idosos, com déficit visual significativo, tendo os pacientes a percepção da natureza irreal de tais alucinações. Objetivo: O presente artigo, tem por finalidade esclarecer as características da sindrome de Charles Bonnet, em especial para os médicos psiquiatras e oftalmologistas, uma vez que tais especialistas tendem a se deparar mais com quadro clínico característico da síndrome. Métodos: Foi realizado pesquisa bibliográfica. A busca de artigos de revisão, bem como relatos de casos foi realizada nos bancos de dados eletrônicos das bases da Latindex, SCIELO e Google Scholar. Revisão da literatura: Com prevalência baixa, a sindrome de Charles Bonnet é em muitos casos subdiagnosticada, tendo em vista que a patologia é pouco conhecida. Seu quadro clínico pode induzir o profissional da saúde a realizar um diagnóstico psiquiátrico errôneo. Uma vez diagnosticada, ofertar tratamento eficaz, que se baseia na correção do quadro oftalmólogicoé de total importância para o sucesso terapêutico do paciente. Conclusão: Após análise da literatura, evidenciou-se que apesar de ter sido descrita pela primeira ver no século XVIII, a sindrome é pouco conhecida. Saber identificar pacientes com o possível quadro, torna-se tarefa importante na busca de ofertar aos mesmos, tratamento adequado.
\end{abstract}

Descritores: Alucinações, Transtornos da visão, Saúde mental

1. Interno do $6^{\circ}$ ano do Curso de Medicina da Universidade São Francisco

2. Médico Oftalmologista da Universidade Federal de São Paulo (UNIFESP) e da Irmandade da Santa Casa de Misericórdia de São Paulo

3. Médico graduado pela Faculdade de Ciências Médicas de Minas Gerais. Psicólogo pela Universidade Federal de São João Del Rei

Trabalho realizado: Universidade São Francisco. Curso de Medicina

Endereço para correspondência: Luiz Guilherme M. Matheus. Av. São Francisco de Assis. SN. Cidade Universitária. Bragança Paulista-SP - Brasil.E-mail: l.g.matheus@outlook.com

\begin{abstract}
Introduction: Charles Bonnet syndrome is defined as the perception of complex visual hallucinations in elderly patients with significant visual impairment, and patients perceive the unreal nature of such hallucinations. Objective: The purpose of this article is to clarify the characteristics of the Charles Bonnet syndrome, especially for psychiatrists and ophthalmologists, since these specialists tend to come up against the clinical picture of the syndrome. Methods: A bibliographic research was carried out. The search for review articles as well as case reports was carried out in the electronic databases of the databases of Latindex, SCIELO and Google Scholar. Literature review: Charles Bonnet syndrome is often underdiagnosed with low prevalence, given that the condition is poorly understood. Its clinical picture may induce the health professional to make an erroneous psychiatric diagnosis. Once diagnosed, offering effective treatment, which is based on the correction of the ophthalmological frame is of total importance for the therapeutic success of the patient. Conclusion: After analyzing the literature, it was evidenced that although it was first described in the eighteenth century, the syndrome is little known. Knowing how to identify patients with the possible picture becomes an important task in the search to offer them the appropriate treatment.
\end{abstract}

Key words: Hallucinations, Vision disorders, Mental health

\section{Introdução}

A Síndrome de Charles Bonnet é uma doença de prevalência incerta observada em sua maioria em pacientes idosos e com déficit visual severo. Com frequência o diagnóstico da síndrome é realizado de forma errada. Em muitas situações, classifica-se o paciente com alucinações provenientes da síndrome de Charles Bonnet, como portador de alguma desordem psiquiátrica. A síndrome caracteriza-se por alucinações visuais complexas em sua maior parte, onde o paciente apresenta crítica da não realidade das mesmas, bem 
como é livre de transtornos cognitivos e psiquiátricos que possam justificar a mesma ${ }^{(1)}$.

\section{Objetivo}

O objetivo desta revisão é evidenciar através do entendimento da Síndrome de Charles Bonnet a importância do seu conhecimento e a possibilidade de realização de diagnósticos mais precisos, em especial por parte do médico psiquiatra e oftalmologista, uma vez que a principal queixa do paciente é a presença de alucinações visuais complexas. Diagnosticar de forma correta tais pacientes é de suma importância para o sucesso terapêutico do mesmo.

\section{Metodologia}

Para a presente revisão foram pesquisados artigos de revisão, bem como relatos de casos nos bancos de dados eletrônicos das bases da Latindex, SCIELO e Google Scholar. Para a busca, usou-se as palavras chaves alucinações, cegueira, transtornos da visão, em língua portuguesa e inglesa. Foi critério de exclusão artigos publicados há mais de 20 anos.

\section{Revisão da Literatura}

A Síndrome de Charles Bonnet foi descrita pela primeira vez no século XVIII, por um filósofo e naturalista suíço, Charles Bonnet. Em 1938 Monsier propôs dar o nome a síndrome aquele que descreveu primeiramente suas características. Bonnet nasceu em Genebra em 13 de março de 1720 e descreveu em seu Essai Analytique surles Facultés de l'Âme (1769) a doença em questão após notar em seu avô, Charles Lullin, sintomas da hoje nomeada síndrome de Charles Bonnet. Bonnet notou que seu avô, um magistrado de 87 anos, apresentava com frequência alucinações visuais complexas, visualizando crianças, pássaros, prédios e tapeçarias, além de cores vívidas. As alucinações eram observadas por Lullin que apresentava na ocasião um deficit visual grave devido a uma catarata em ambos os olhos. Lullin, não apresentava antecedente de doenças psiquiátricas bem como cognitivas que pudessem corroborar para os quadros alucinatórios ${ }^{(1-2)}$.

Mais tarde em sua vida, o próprio Charles Bonnet experimentou alucinações visuais tidas por ele próprio como irreais. Sabe-se a esse respeito que Bonnet teve um quadro grave de baixa da acuidade visual, não havendo mais detalhes nos registros do motivo de tal quadro oftalmológico ${ }^{(3)}$.

A doença aparentemente apresenta baixa prevalência, mas admite-se que não existam dados precisos, em parte pelo desconhecimento dos médicos em diagnosticar a doença e em parte devido ao receio dos pacientes de relatarem tais alucinações aos seus médicos, justificado pelo estigma de serem considerados doentes mentais ${ }^{(3-4)}$. Um estudo, entretanto, com pacientes portadores de glaucoma evidenciou que 11 pacientes, $12,3 \%$ do total analisado no estudo apresentavam a síndrome de Charles Bonnet. A pesquisa não evidenciou diferença na prevalência entre os sexos e observou aumento na prevalência com o aumento da idade dos pacientes e com a presença de baixa acuidade visual ${ }^{(5)}$.

As alucinações vivenciadas pelos pacientes acometidos com a síndrome de Cherles Bonnet são caracterizadas como complexas em sua grande maioria. Os padrões podem variar, sendo relatados casos de imagens coloridas sem uma forma definida até imagens de veículos ou pessoas, por exemplo. Alguns pacientes, consideram relatar a existência de alucinações auditivas associadas as alucinações visuais. Entretanto tal associação mostra-se controversa no entendimento de alguns autores. Vale salientar aqui que Teunisse em seus critérios para o diagnóstico da síndrome de Charles Bonnet coloca a ausência de outros tipos de alucinações como critério para firmar o diagnóstico ${ }^{(4)}$. De fundamental importância nota-se o fato dos pacientes terem crítica sobre a irrealidade das alucinações que experimentam, evidenciando de certa forma e afastando os quadros psiquiátricos muitas vezes associados a tais percepções.

Ainda não se conhece totalmente a fisiopatologia da doença. O que algumas teorias postulam em concordância é o fato das alucinações serem produzidas no córtex visual e seu conteúdo ser dependente da região que as mesmas foram originadas, por exemplo: estudo realizado por Santhouse et al, 2000 $0^{(6)}$ evidenciou que pacientes que alucinavam com objetos e movimentos, teriam comprometimento em região de córtex occipito temporal ventral. Dessa forma, acredita-se que com a queda da acuidade visual ocorre uma baixa estimulação cortical, e a partir daí, existe uma tentativa de reorganização anatômica, gerando um desequilíbrio fisiológico nas reações neuronais. Com isso algumas áreas ficam hiper excitadas, levando a ativação de campos ectópicos e autônomos, gerando as alucinações próprias da síndrome ${ }^{(7)}$. A teoria da desaferentação, situação semelhante a que ocorre em pacientes com membros amputados gerando a síndrome do membro fantasma, é sustentada baseado em um estudo que selecionou 13 pacientes com visão normal, onde os mesmos ficaram com os olhos vendados por cinco dias. Dos 13 pacientes do grupo, 10 relataram a presença de alucinações visuais após um dia em média ${ }^{(4)}$. Fica evidente que a fisiopatologia da Síndrome de Charles Bonnet ainda carece de mais estudos para seu real e completo entendimento.

Com relação ao diagnóstico da doença, deve-se 
saber que o mesmo não é simples, uma vez que as alucinações se apresentam muitas vezes com variações em seu conteúdo, duração e frequência. Teunisse propôs alguns critérios que são hoje os mais utilizados para firmar o diagnóstico da Síndrome de Charles Bonnet. São eles:

1) Alucinações visuais complexas persistentes e repetitivas

2) Consciência autocrítica das alucinações

3) Ausência de alucinações de outras modalidades sensoriais

4) Ausência de ideação delirante sobre o conteúdo das alucinações ${ }^{(8)}$.

É frequente o paciente ser diagnosticado incialmente de forma errônea, sendo muitas vezes tratado como portador de esquizofrenia ou psicose tardia. Para tanto é importante fazer diagnóstico diferencial com várias patologias que podem causar alucinações visuais. Patologias psiquiátricas devem ser descartadas como os quadros de transtorno bipolar e doenças neurológicas como a doença de Parkinson e epilepsia. Lesões cerebrais, uso de drogas e fármacos também devem ser investigadas quando a queixa de alucinações surge em um paciente ${ }^{(8-9)}$.

O tratamento da síndrome baseia-se fundamentalmente no esclarecimento da doença pelo paciente, explicando ao mesmo que não se trata de uma doença mental. Tal elucidação ajuda na busca e na confiança do paciente para tratar o quadro apresentado. É imprescindível o acompanhamento dos sintomas de temor, ansiedade gerados pelas alucinações e pela incerteza de diagnóstico e prognóstico do paciente. O seguimento oftalmológico deve ser buscado de forma imediata, para reverter, quando possível a baixa acuidade visual desses pacientes, motivo principal de suas alucinações. As alucinações muitas vezes cessam quando o paciente adquire cegueira total.

Ressalta-se que alguns pacientes tiveram melhora dos quadros de alucinações visuais com o uso de carbamazepina, tendo em vista que esta droga tem a capacidade de diminuir o turnover do ácido gama-aminobutírico e aumentar o turnover do glutamato, com isso, provavelmente inibe de forma importante os circuitos neuronais que se encontram hiper excitados responsáveis pelas alucinações visuais características da doença. Existe, entretanto, a necessidade de mais estudos para firmar o benefício e o mecanismo exato da medicação na síndrome de Charles Bonnet, tendo em vista que existe falta de relatos na literatura sobre mais detalhes da resposta de tais pacientes ao uso de antipsicóticos $^{(10-13)}$.

\section{Conclusão}

A síndrome de Charles Bonnet é um quadro que é subdiagnosticado e que deve ser suspeitado em todo paciente idoso que apresenta baixa acuidade visual e alucinações visuais, com crítica presente. Deve ser descartado sempre nos pacientes outras comorbidades que possam justificar a sintomatologia apresentada, sendo de base psiquiátrica e/ou neurológica. O conhecimento por parte dos médicos, em especial do médico psiquiatra e oftalmologista é de suma importância para um diagnóstico mais preciso e um tratamento eficaz.

\section{Referencias}

1. Maranhão Filho PA. Síndrome de Charles Bonnet: a propósito de um caso. Rev Bras Neurol. 2009: 45(2):21-4.

2. Ruiz Medrano J, Santos-Bueso E, García-Sánches J. Charles Bonnet and his theories. Arch Soc Esp Oftalmol. 2013: 88 (10):68-9.

3. Berrios G, Brook P. A síndrome de Charles Bonnet e o problema dos transtornos de percepção visual nos idosos. Rev Latinoam Psicopatol Fundam. 2011; 14(3):530-41.

4. Jackson ML, Ferencz J. Charles Bonnet Syndrome: visual loss and hallucinations. CMAJ. 2009: 181(3-4):175-6.

5. Freiman T M, Surges R, Vougioukas V I, Hubbe U, Talazko J, Zentner J, et al. Complex visual hallucination (Charles Bonnet Syndrome) in visual field defects following cerebral surgery. Report of four cases. J Neurosurg. 2004: 101(5):846-53.

6. Santhouse AM, Howard RJ, ffytche DH. Visual hallucinatory syndromes and the anatomy of the visual brain. Brain. 2000; 123 (Pt 10):2055-64.

7. Cortizo V, Rosa AAM, Soriano DS, Takada LT, Nitrini R. Síndrome de Charles Bonnet: alucinações visuais em pacientes com doenças oculares - Relato de Caso. Arq Bras Oftalmol 2005; 68 (1):129-32.

8. Muñoz Cortez H, Vargas Rueda A. Síndrome de Charles Bonnet: revisión de tema. Rev. Colomb. Psiquiatr. 2007: 36(2):292-306.

9. Rojas-Rojas H, Borja-Ballesteros C, Escobar-Córdoba F. Síndrome de Charles Bonnet: presentación de dos casos. Rev Chil Neuro-Psiquiatr. 2007; 45(2):161-5.

10. Pérez Longás B, Pomares Martínez T, Pedrós Roselló A. Psicosis de inicio tardío y síndrome de Charles Bonnet: dificultades diagnosticas. A proposito de un caso. Psiquiar Biol 2007:24(2):67-9.

11. Eperjesi F, Akbarali N. Rehabilitation in Charles Bonnet syndrome: a review of treatment options. Clin Exp Optom. 2004; 87(3):149-52.

12. Mitra S, Khanra S, Mondal S K, Kavoor AR, Das B. Carbamazepine in treatment of visual hallucinations: a case of chronic hallucinatory psychosis. Indian J Psychol Med. 2015: 37(4): 449-51.

13. Segers K. Charles Bonnet syndrome disappearing with carbamazepine and valproic acid but not with levetiracetam. Acta Neurol Belg. 2009: 109(1):42-3.

Trabalho recebido: $23 / 11 / 2017$

Trabalho aprovado: 29/03/2018 